\title{
APPLICATION OF A NEW METHOD FOR CALCULATION OF LOW-FREQUENCY WAVE VECTORS
}

\author{
S. M. Hatch*, and J. W. LaBelle*
}

\begin{abstract}
The problem of resolving spatial and temporal properties of waves, so-called "space-time ambiguity", is a longstanding issue of single-spacecraft measurements. The general case can be insoluble, but in special cases in which certain assumptions hold, such as when each frequency corresponds to a single wave vector, the ambiguity can be resolved. Recently a method has been proposed to obtain wave vectors from single-spacecraft measurements of Alfvén wave-modulated magnetic fields and currents [Bellan, 2016], through application of the Wiener-Khinchin theorem to cross-correlation of the current density $\mathbf{J}$ and magnetic field $\mathbf{B}$, and to the autocorrelation of $\mathbf{B}$. We apply this method to spacecraft data, obtained by culling, from a large database of inertial Alfvén waves observed by the FAST satellite, two case study intervals during which extraordinarily large modulated currents were measured by the FAST particle detectors in burst mode. Results of this analysis for at least one of the two case studies appear consistent with known properties of ionospheric inertial Alfvén waves and pass error and consistency checks within the analysis method.
\end{abstract}

\section{Introduction}

Waves and turbulence play an important role in many space plasma processes, such as particle acceleration, heating, and scattering in many different environments. Understanding the wave-particle and wave-wave interactions in these processes requires thorough measurements of the wave modes, meaning not only the wave frequency but also the wave vectors. While frequencies are relatively straightforward to measure, wave vectors present a challenge. A variety of techniques have been applied, usually involving spatially separated measurements on one or more spacecraft as reviewed by LaBelle and Kintner [1989] and updated by Pfaff and Marionni [1998].

Single-point techniques for inferring wavelength are sparse. In some cases, wherein an instrument measurement is effectively an average over a region of space as in the case of the

*Department of Physics and Astronomy, Dartmouth College, Hanover, New Hampshire, USA 
double-probe electric field experiment, finite--wavelength effects affect the measurement in ways that can be used to obtain information about the wave vectors; examples are given in the references above. In other cases, in which the spacecraft velocity exceeds all phase velocities of modes in the system, the Taylor "frozen-in" hypothesis applies and allows the wavenumber spectrum to be inferred from the frequency spectrum observed on a single spacecraft, though such interpretations hold only under restricted conditions, as pointed out in a famous warning issued by Fredericks and Coroniti [1976]: “... interpretations of the computed [frequency] spectrum in terms of the rest frame [wave vector] spectrum are ambiguous, can indeed lead to erroneous physical conclusions, and thus represent a procedure fraught with pitfalls and dangers for those who employ it."

Recently, Bellan [2016] proposed a new technique for estimating the wave vector of lowfrequency (e.g., Alfvén or ion cyclotron) waves for which the corresponding current density is divergence free, resolving space-time ambiguity through independent measurements of current density $\mathbf{J}$ and magnetic field $\mathbf{B}$ at the same location. The technique relies on the condition that each frequency component in the spacecraft frame corresponds to a unique wave vector. Until recently, this technique was generally impractical due to the difficulty of direct particle measurements of fluctuating currents with sufficient cadence to resolve Alfvén waves and sufficient counting statistics to overcome noise. However, some recent experiments, including for example the FAST satellite, include particle measurements that meet this criterion for at least some large amplitude events.

In this paper, we apply the Bellan [2016] technique to real spacecraft data. (Bellan [2016] applied the technique to synthetic data; to the knowledge of the authors, the only other study that has applied this technique to spacecraft data was performed by Gershman et al. [2017] using Magnetospheric Multiscale observations.) Section 2 below, based largely on the work of Bellan [2016] (hereafter B16), describes the technique and methods of testing it, and Section 3 presents FAST satellite data and describes attempts to apply the B16 method to two intervals of large amplitude inertial Alfvén waves in the acceleration region. Section 4 concludes with comments on the plausibility of the analysis results, and suggestions of means by which the method could be further validated.

\section{Methodology}

The B16 method is based on Ampère's law, which via plane--wave decomposition may be written in wave vector and frequency space as

$$
\mu_{0} \mathbf{J}(\omega)=i \mathbf{k}(\omega) \times \mathbf{B}(\omega) .
$$

Two important assumptions are obvious. The first is that the displacement current $\epsilon_{0} \partial \mathbf{E} / \partial t$ is neglected. The second, fairly restrictive, assumption is that there is a unique $\mathbf{k}$ for each $\omega$. Taking the cross product of each side of Equation (1) with $\mathbf{B}^{*}(\omega)$ and applying

$\mathbf{k}(\omega) \cdot \mathbf{B}(\omega)=0$ yields the primary result:

$$
\mathbf{k}(\omega)=i \mu_{0} \frac{\mathbf{J}(\omega) \times \mathbf{B}^{*}(\omega)}{\mathbf{B}(\omega) \cdot \mathbf{B}^{*}(\omega)} .
$$


In practice these quantities are calculated via application of the Wiener-Khinchin theorem to cross-correlation of $\mathbf{J}$ and $\mathbf{B}$, and to auto-correlation of $\mathbf{B}$,

$$
\mathbf{k}_{\mathrm{calc}}(\omega)=i \mu_{0} \frac{\int_{0}^{T} \mathbf{H}(\tau) e^{-i \omega \tau} d \tau}{\int_{0}^{T} F(\tau) e^{-i \omega \tau} d \tau},
$$

where $\mathbf{H}(\tau)=\langle\mathbf{J}(t) \times \mathbf{B}(t+\tau)\rangle, F(\tau)=\langle\mathbf{B}(t) \cdot \mathbf{B}(t+\tau)\rangle$, and brackets represent time averages. These quantities can be estimated from discrete calculations provided time series of appropriate components of $\mathbf{J}$ and $\mathbf{B}$ are measured by a single spacecraft in space, at the same location (or with spatial separation small compared to the wavelength).

Bellan [2016] proposes several consistency checks for solutions obtained from Equation (3), which in turn test the validity of the underlying assumptions. The simplest of these,

$$
\langle\mathbf{J}(t) \times \mathbf{B}(t)\rangle=0,
$$

is a consequence of the so-called "reality conditions" $\mathbf{B}^{*}(-\omega)=\mathbf{B}(\omega)$ and $\mathbf{k}(-\omega)=-\mathbf{k}(\omega)$ [Bernstein and Engelmann, 1966; Schmidt, 1979]. In discrete analysis using spacecraft data Equation (4) becomes

$$
\langle\mathbf{J}(t) \times \mathbf{B}(t)\rangle_{\text {norm }}=\frac{1}{N} \frac{\sum_{n=1}^{N} \mathbf{J}[n] \times \mathbf{B}[n]}{\sum_{n=1}^{N}|\mathbf{J}[n]||\mathbf{B}[n]|},
$$

where $N$ is the total number of measurements of $\mathbf{J}$ and $\mathbf{B}$, and the reality conditions are approximately satisfied if $\left|\langle\mathbf{J}(t) \times \mathbf{B}(t)\rangle_{\text {norm }}\right| \ll 1$.

Additionally, the assumption that each frequency component is associated with a unique $\mathbf{k}$ may be tested by insertion of $\mathbf{k}_{\text {calc }}$ from Equation (3) into Ampère's law (Equation (1)), which yields a predicted current $\mathbf{J}_{\text {pred }}(\omega)=i \mathbf{k}_{\text {calc }}(\omega) \times \mathbf{B}(\omega)$. Using $\mathbf{J}_{\text {pred }}$ and the observed $\mathbf{J}$, Bellan [2016] defines respectively the error angle and the relative magnitude error

$$
\begin{aligned}
\theta_{\text {err }}(\omega) & =\cos ^{-1}\left(\operatorname{Re}\left(\mathbf{J}_{\text {pred }} \cdot \mathbf{J}\right) /\left|\mathbf{J}_{\text {pred }}\right||\mathbf{J}|\right) ; \\
M_{\text {err }}(\omega) & =|| \mathbf{J}_{\text {pred }}|-| \mathbf{J}_{\text {pred }}|| /\left(\left|\mathbf{J}_{\text {pred }}\right|+\left|\mathbf{J}_{\text {pred }}\right|\right) ;
\end{aligned}
$$

where $\theta_{\text {err }} \in[0, \pi]$ and $M_{\text {err }} \in[0,1]$. If $\mathbf{k}(\omega)$ is unique, $\theta_{\text {err }}(\omega) \ll \pi$ and $M_{\text {err }}(\omega) \ll 1$. On the other hand, in the presence of more than one $\mathbf{k}$ (e.g., standing waves or two or more waves from distinct source regions) one or both error quantities are large and Equation (3) is not valid [Bellan, 2016].

The wave vector $\mathbf{k}$ in Equation (3) and the diagnostic quantities in Equations (5)-(6) can be calculated from independent measurements of $\mathbf{B}$ and $\mathbf{J}$ at a single location in space (i.e., by a single spacecraft). All three components of $\mathbf{B}$ are measured with fluxgate magnetometers on many spacecraft, often with sub-nT resolution, allowing the modulated magnetic field of reasonably large amplitude Alfvén waves to be detected. The resolution 
of the fluxgate magnetometer aboard FAST, for example, was 2 nT [Carlson et al., 2001], and the effective maximum sample rate (limited by recursive filtering) was $128 \mathrm{~S} / \mathrm{s}$ in "burst mode", which was triggered in real time based on survey-mode observations.

Relative to measurement of the magnetic field, an independent measurement of the modulations of particle currents associated with the Alfvén wave is much more problematic. (Although current is often inferred from the time derivative of the appropriate magnetic field component, valid under certain assumptions, such an estimate obviously does not provide what is needed for the B16 analysis.) The particle currents must be calculated from measured electron and ion distribution functions, either via on-board electronics or during post processing, which poses two formidable challenges: first, the cadence of distribution function measurements, which often requires accumulation of particle counts in various instrument geometries relative to $\mathbf{B}_{0}$, is usually too slow to sample Alfvénic current modulations; second, even if the measurement cadence is sufficiently high (or perhaps because it is so high for detectors with modest geometric factors), the accumulated counts corresponding to various components of the particle distribution functions may be too low for the modulations associated with Alfvén waves to be observable above the statistical counting uncertainty. For these reasons the identification of intervals when the B16 technique may be applied to satellite data is nontrivial.

The FAST satellite frequently operated in burst mode, yielding a large database of electron and ion distributions measured by ion and electron electrostatic analyzers (ESAs) at $\sim 12.8 \mathrm{~S} / \mathrm{s}$ [Carlson et al., 2001], which in principle is high enough to resolve Alfvén waves up to $\sim 6 \mathrm{~Hz}$ in the spacecraft frame. The remaining issue, identification of time intervals when there is a chance to resolve Alfvén wave-modulated currents, is addressed in the next section.

\section{Data presentation}

In extensive studies of storm-time and IMF effects on wave energy transport and deposition, Hatch et al. [2016; 2017; 2018] developed an algorithm for identification of inertial Alfvén waves (IAWs) observed by FAST while operating in "fast survey" mode. The algorithm, which is derived from that employed by Chaston et al. [2003; 2007], begins by examining the slope of the cross-track (approximately east-west) component of the magnetic field measured by the fluxgate magnetometer to obtain an estimate of the field-aligned current $J_{\text {mag. }}$. Large amplitude fluctuations $\left(\left|J_{\text {mag }}\right|>10 \mu \mathrm{A} / \mathrm{m}^{2}\right)$ are then identified and subjected to various threshold criteria for modulations of the magnetic field and electric field and of the current derived ESA measurements $J_{\mathrm{ESA}}$, as well as a requirement that the ratio of field fluctuations transverse to $\mathbf{B}_{0}, E_{\perp} / B_{\perp}$, be within an order of magnitude of the local Alfvén speed [e.g., Keiling, 2009]. Applying these criteria to all FAST observations in 1996-1999 yields a database of more than 160,000 IAW observations; a subset of 42,500 observations are coincident with burst-mode $\left(f_{S} \simeq 12.8 \mathrm{~S} / \mathrm{s}\right)$ ESA particle measurements. These are the events for which it is theoretically possible to measure particle current modulations of frequencies up to $6 \mathrm{~Hz}$.

The IAW database is searchable based on various parameters, including the amplitudes of 
$J_{\text {mag }}$ and $J_{\mathrm{ESA}}$ and the times and durations of each IAW. It is therefore possible to search for streaks of IAW observations with a combination of 1) largest modulated currents, using either $J_{\text {mag }}$ or $J_{\mathrm{ESA}}$, and 2) longest contiguous period of IAW observations. Of all 42,500 burst-mode observations, there are only a few streaks lasting at least $10 \mathrm{~s}$ with a gap of no more than $2 \mathrm{~s}$ between subsequent observations. Following this procedure we have selected two periods that appear suitable for application of the B16 method: 14:50:56-14:51:06 UT during orbit 9585 on Jan. 23, 1999; and 11:32:52-11:33:09 during orbit 9627 on Jan. 27, 1999.

\subsection{Jan. 23, 1999 observations}

Figure 1 shows a 40-s interval during which FAST was above $77^{\circ}$ ILAT in the southern hemisphere cusp/cleft region, and observed strongly field-aligned electrons (Figure 1b), transversely accelerated ionospheric ions (Figure 1d) and electric field fluctuations regularly exceeding $50 \mathrm{mV} / \mathrm{m}$ (Figure $1 \mathrm{~g}$ ). These modulations were identified as IAWs by the algorithm described above. During the preceding hour the interplanetary magnetic field (IMF) clock angle $\phi_{\text {IMF }}$ was strongly duskward, with $\left\langle\mathbf{B}_{\text {IMF }}\right\rangle=\langle-1.0,10.8,4.2\rangle \mathrm{nT}$ in GSM coordinates. Large IMF $B_{y}$ conditions favor longitudinal bifurcation of the cusp [Massetti, 2005]. Statistical studies of FAST data under these conditions suggest that these observations may have taken place in the vicinity of what has been termed the "Alfvénic cusp" [Hatch et al., 2017].

Within the $\sim 11$ s following 14:50:56 UT, indicated by vertical red lines in Figure 1, a series of very large current density modulations $\left(>50 \mu \mathrm{A} / \mathrm{m}^{2}\right)$ were directly measured by the particle instruments (Figure 1e) and indirectly measured by deflections in the crosstrack magnetic field component (Figure 1f). Figure 1e shows particle currents parallel to the background magnetic field. It is evident that the total particle current (black trace) is dominated by precipitating electrons (red trace) rather than upflowing ions (green trace). The current exhibits large modulations with an approximate 1-s timescale. Similar modulations are observed in the current derived from the magnetic field, and at some instances the directly and indirectly measured current modulations appear correlated. As expected in the presence of IAWs, the bottom panel (Figure 1g) shows large-scale modulations of the electric field component measured along the spacecraft trajectory, which is roughly north-south.

For the 11-s period 14:50:56-14:51:07 UT (vertical red bars, Figure 1), Figure 2 shows an expanded view of FAST data required as inputs for B16 analysis. The top left panels show components of the magnetic field measured by the fluxgate magnetometer and transformed to the field-aligned coordinate system most closely aligning with geomagnetic north $\left(B_{x}\right.$, top left panel) and geomagnetic east ( $B_{y}$, center left panel). The bottom left panel shows the total current density along the magnetic field directly measured by the electron and ion ESAs $\left(J_{z}\right.$, same quantity as plotted in Figure 1e). Because ESA measurements of particle distributions are confined to the spacecraft spin plane it is not possible to fully measure $J_{x}$ or $J_{y}$ in this analysis; however, the perpendicular components of the current modulations associated with the Alfvén waves, $\mathbf{J}_{\perp}$, are expected to be small. (Over this interval $\left|\mathbf{J}_{\perp}\right| \leq 10 \mu \mathrm{A} / \mathrm{m}^{2}$, and its inclusion does not alter results of the present analysis or 


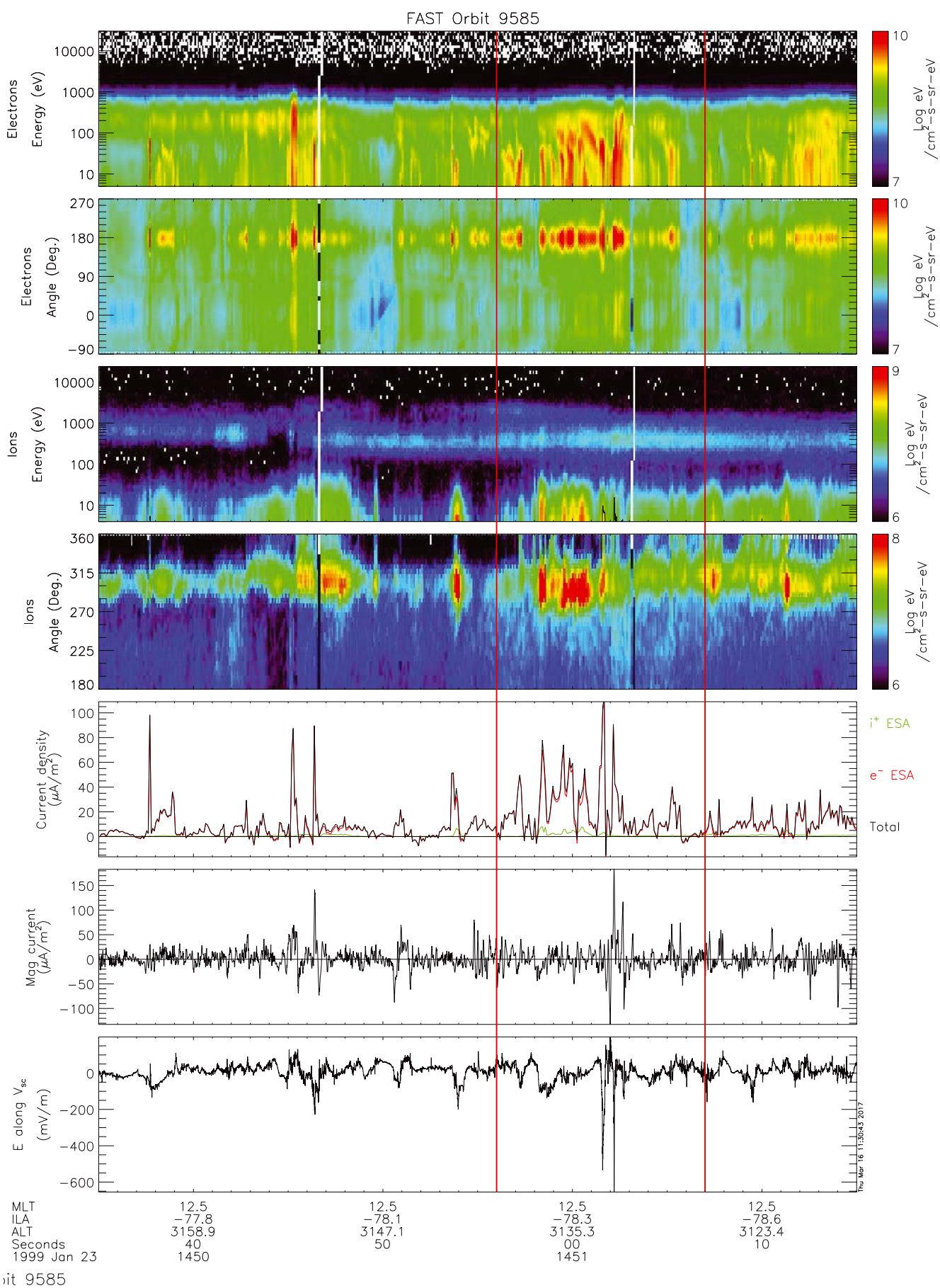

Figure 1: FAST satellite observations in the southern hemisphere cusp region of a series of field-aligned electron bursts (Figures 1a-b), transversely accelerated ions (Figures 1c-d), and strongly modulated field-aligned currents (Figures 1e-f) and electric fields (Figure 1g) identified as a train of IAW observations via the Hatch et al. [2016] algorithm.

the corresponding conclusions.) Setting $J_{x}=J_{y}=0$ in Equation (2) implies both that $\mathbf{k}$ does not depend on $B_{z}$, which is therefore not used as an input, and that the field-aligned component of the wave vector $k_{z}$ cannot be calculated in the present analysis. 


\section{Inputs}
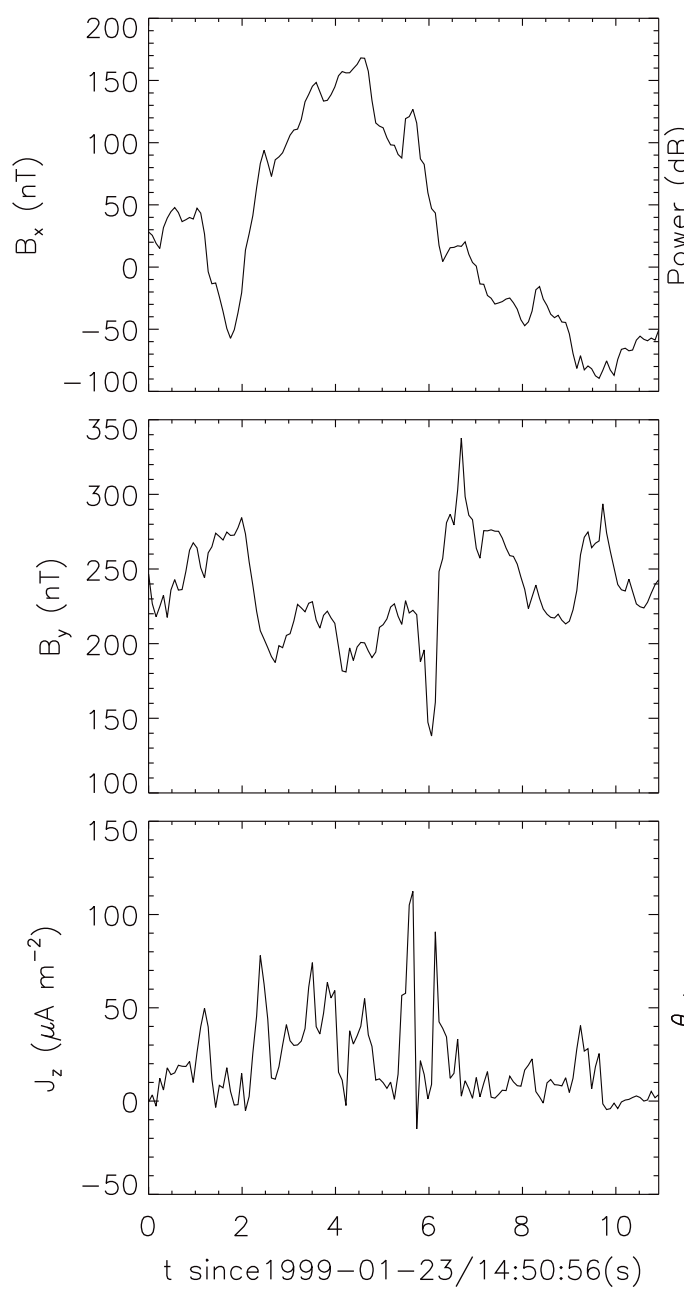

Orbit 9585
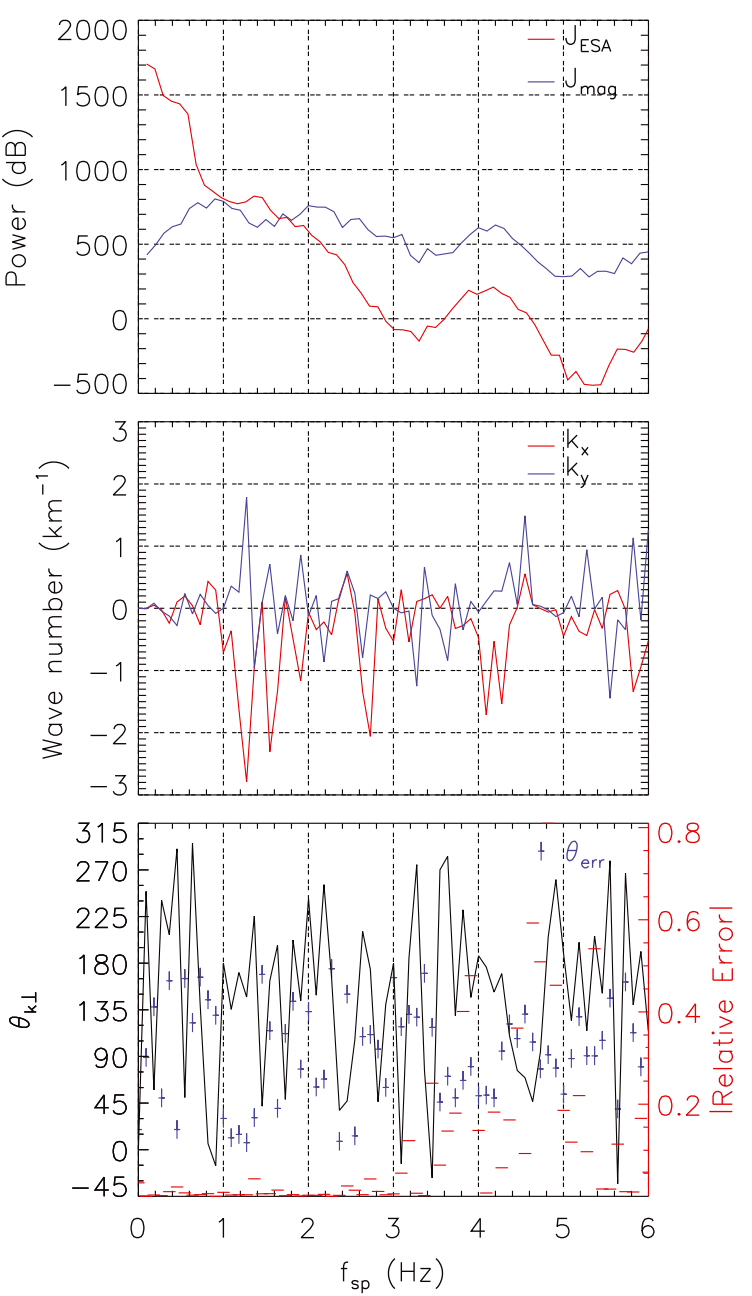

Figure 2: B16 analysis magnetic field and current density inputs (left column) and corresponding Fourier spectra (top panel, right column), calculated wave number spectra (center panel, right column), and diagnostics (bottom panel, right column) for the 11-s period between red bars in Figure 1.

The top right panel of Figure 2 shows two power spectra computed with the Slepian [1978; 1983] multitapering technique: directly measured current modulations $J_{\mathrm{ESA}}$ (red trace, same as $J_{z}$ ) and those indirectly measured via the spatial derivative of the cross-track magnetic field $J_{\text {mag }}$ (blue trace), where the time series of the latter has been averaged and decimated by a factor of 10 in order to match the ESA sampling rate and Nyquist frequency, approximately $12 \mathrm{~Hz}$ and $6 \mathrm{~Hz}$, respectively. Both spectra show a broad peak at $1-2 \mathrm{~Hz}$ and another slightly above $4 \mathrm{~Hz}$. The peak near $1 \mathrm{~Hz}$ corresponds to the $\sim 1-\mathrm{s}$ timescale of the modulations visible in the $J_{z}$ time series (bottom left panel of Figure 2). The $4-\mathrm{Hz}$ peak is considerably better expressed in the current derived from the fluxgate magnetometer, possibly in consequence of the superior time resolution of magnetometer data. These peaks suggest the frequency ranges in which the B16 analysis is likely to 
yield meaningful results.

The bottom right panels of Figure 2 present the results of B16 analysis as a function of spacecraft-frame frequency $f_{s p}$ : the calculated wave number spectra $k_{x}$ and $k_{y}$ (center panel, red and blue traces, respectively) and the angle of perpendicular wave propagation $\theta_{k_{\perp}}=\tan ^{-1}\left(\frac{k_{y}}{k_{x}}\right)$ (black trace, bottom panel), where $\theta_{k_{\perp}}=0$ corresponds to geomagnetic north. The bottom panel also shows the error parameters described in Section 2, $\theta_{\text {err }}$ (blue crosses) and $M_{\text {err }}$ (red dashes), which are indicators of the degree to which the assumption holds that a unique $\mathbf{k}$ exists at each frequency [Bellan, 2016].

Examining the bottom right panel reveals two frequency ranges, $1-1.5 \mathrm{~Hz}$ and $4-4.5 \mathrm{~Hz}$, in which the error parameters take on relatively low values. In the first frequency range, $M_{\mathrm{err}} \simeq 0$ and $\theta_{\mathrm{err}}=10-15^{\circ}$. In the second, $M_{\mathrm{err}} \leq 0.2$ and $\theta_{\mathrm{err}} \simeq 45^{\circ}$; each is larger than in the first range but smaller than most values across the rest of the spectrum. These frequency ranges correspond to the two peaks evident in each power spectrum (top right panel, Figure 2) where it is expected that the spectral inputs are dominated by real current fluctuations rather than noise.

Within these two frequency ranges $k_{x}$ takes on values between -2.8 and and $-0.5 \mathrm{~km}^{-1}$, with $k_{y}$ values between 0.5 and $1.8 \mathrm{~km}^{-1}$ and $\theta_{k_{\perp}} \simeq 160^{\circ}$; outside these frequency ranges $\theta_{k_{\perp}}$ fluctuates wildly and the error parameters are large. The 1.3-Hz peak corresponds to slightly larger $k$ components, which in turn correspond to transverse wavelengths $\lambda_{\perp} \simeq 2-11 \mathrm{~km}$.

The significant evidence in Figures $1 \mathrm{a}$ and $1 \mathrm{~b}$ of Alfvénically accelerated electrons corresponds to a physical requirement $k_{\perp} \lambda_{e} \sim 1$, where $\lambda_{e}=c / \omega_{p e}$ is the electron inertial length, in order for the waves to generate a parallel electric field capable of the observed acceleration [Goertz and Boswell, 1979]. Observations in Figure 1 were made at relatively high altitudes $(>3000 \mathrm{~km})$ where electron densities are typically of order $10-100 \mathrm{~cm}^{-3}$ [Figure 2a in Chaston et al., 2003; Kletzing et al., 1998], implying $f_{p e} \sim 30-90 \mathrm{kHz}$ and $\lambda_{e} \sim 0.5-1.7 \mathrm{~km}$. The wave vectors determined from the B16 analysis therefore seem consistent with the condition $k_{\perp} \lambda_{e} \sim 1$. For example if one assumes $\lambda_{\|}=2 \times 10^{4} \mathrm{~km}$, $n_{e}=30 \mathrm{~cm}^{-3}$, and $V_{\mathrm{A}}=8 \times 10^{3} \mathrm{~km} / \mathrm{s}$, the two-fluid Alfvén wave dispersion relation [e.g., Stasiewicz et al., 2000] predicts $k_{\perp} \lambda_{e}=2.9$. (The ion gyroradius $\rho_{i}$ is about an order of magnitude less than the electron inertial length $\lambda_{e}$ below $\sim 2 R_{E}$, and is neglected in this estimation.)

Checking the reality conditions via Equation (5) over the 11-s period, we obtain $\langle\mathbf{J}(t) \times$ $\mathbf{B}(t)\rangle_{\text {norm }}=\left\langle 5.3 \times 10^{-3}, 1.6 \times 10^{-3}, 0\right\rangle$. The $x$ and $y$ components are significantly below unity, suggesting the condition holds. (The $z$ component is not calculated.)

\subsection{Jan. 27, 1999 observations}

Figure 3 shows a 49-s window of pre-noon southern hemisphere observations near $75^{\circ}$ ILAT on Jan. 27, 1999. During this pass FAST instruments observed strongly fieldaligned electrons (Figure 3b) and transversely accelerated ionospheric ions (Figure 3d). Shortly after the burst of field-aligned electrons at 11:32:45 UT semi-periodic wave forms 


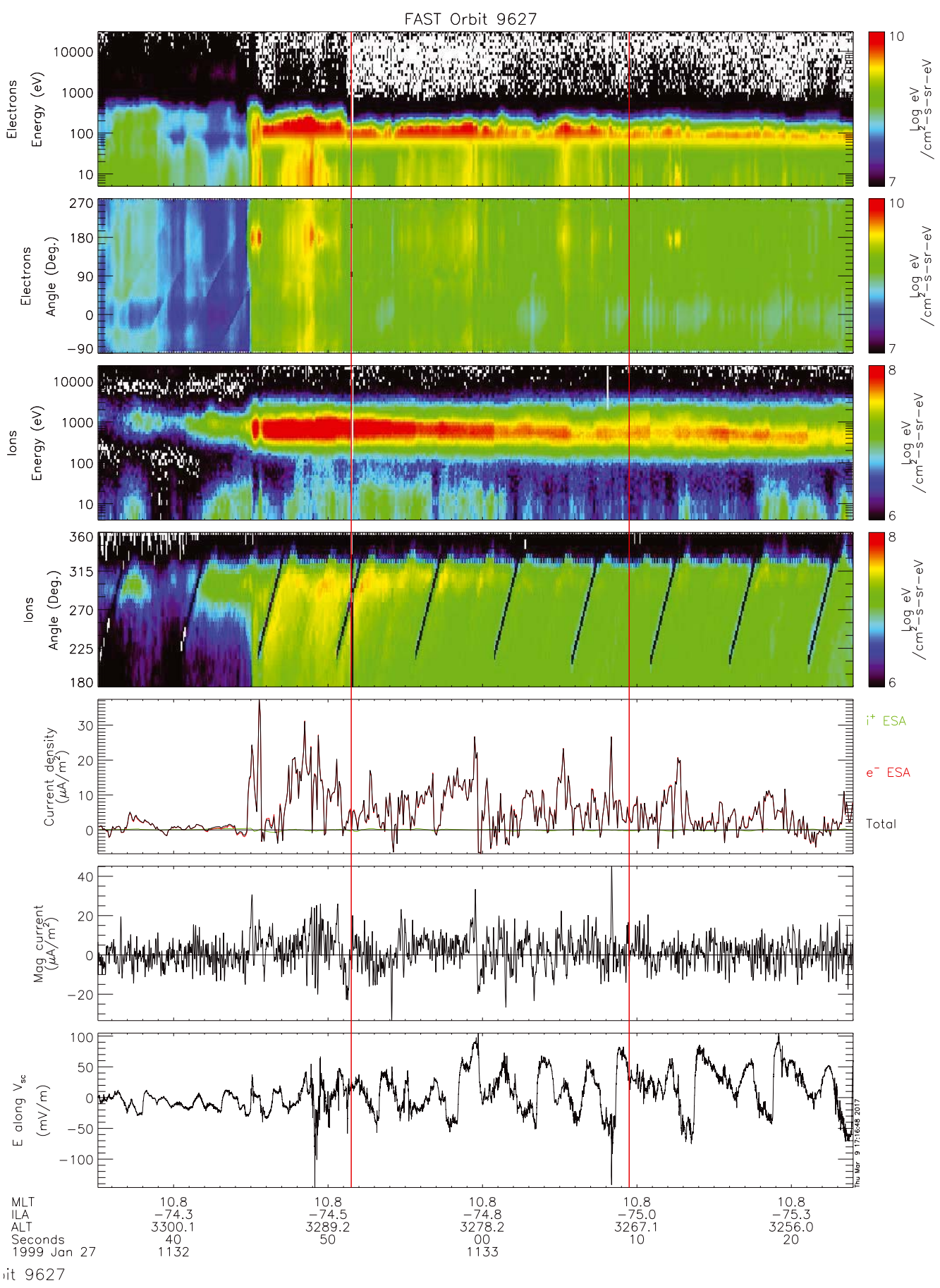

Figure 3: FAST satellite observations in the pre-noon southern hemisphere coincident with a train of IAW observations identified via the Hatch et al. [2016] algorithm, in the same layout as Figure 1.

appear in $J_{\mathrm{ESA}}$ (Figure 3e) and $J_{\text {mag }}$ (Figure 3f), as well as in the component of the electric field measured along the spacecraft trajectory (Figure 3g). For the hour preceding this interval $\phi_{\mathrm{IMF}}$ was steadily dusk-south, with $\left\langle\mathbf{B}_{\mathrm{IMF}}\right\rangle=\langle-4.3,3.9,-5.3\rangle \mathrm{nT}$ in GSM coordinates. These IMF conditions favor the appearance of traveling convection vortices 
in this local time sector [Sandholt et al., 2004].

Inputs
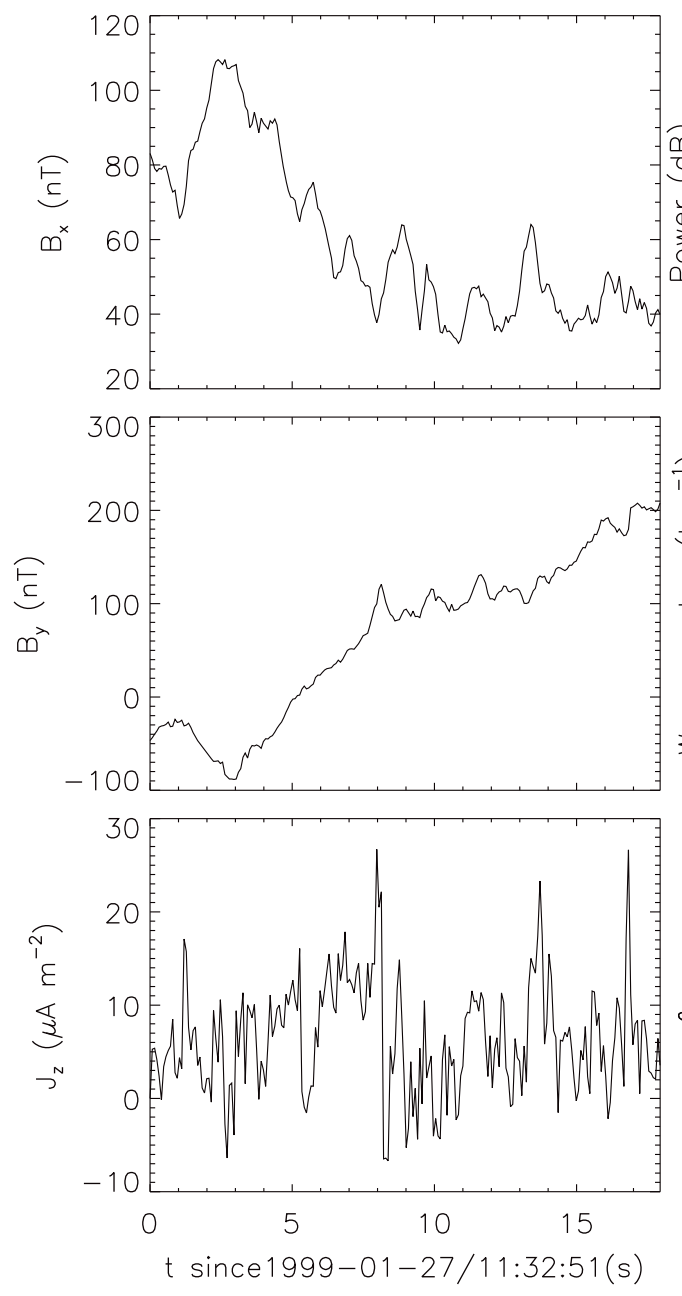

Orbit 9627
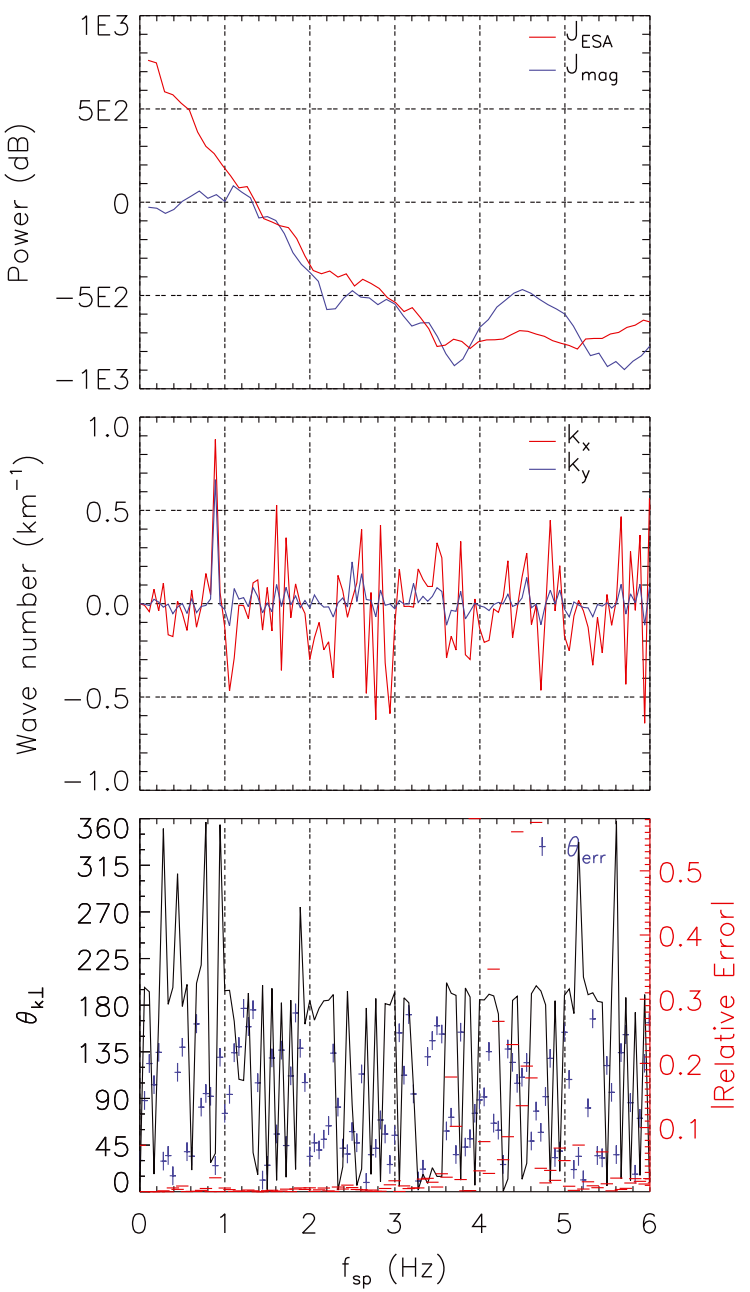

Figure 4: Alfvén wave vector properties inferred from B16 analysis of the 18-s period shown between red bars in Figure 3.

We apply B16 analysis to the 18-s period between the red bars at 11:32:51.5 and 11:33:09.5 UT. Figure 4 shows both the inputs for the analysis and the resulting $\mathrm{k}$ spectra, in the same layout as Figure 2. In descending order, panels in the left column show $B_{x}, B_{y}$, and $J_{z}$, which are used for B16 analysis of this period.

The top right panel of Figure 4 shows power spectra of $J_{\mathrm{ESA}}$ (red trace, same as $J_{z}$ ) and $J_{\text {mag }}$ (blue trace). In contrast to power spectra of these quantities for the previous event (Figure 2, top right panel), which both displayed enhanced power over a small range of frequencies, distinct features are not apparent in these spectra. However, power increases considerably at frequencies below $\sim 2 \mathrm{~Hz}$, consistent with the modulations observable in the time series (bottom left panel, Figure 4).

The two lower panels in the right column of Figure 2 present the results of B16 analysis 
as a function of spacecraft-frame frequency $f_{s p}$ : the calculated wave number spectra $k_{x}$ and $k_{y}$ (center panel, red and blue traces, respectively) and the angle of perpendicular wave propagation $\theta_{k_{\perp}}=\tan ^{-1}\left(\frac{k_{y}}{k_{x}}\right)$ (black trace, bottom panel). The bottom panel also shows $\theta_{\text {err }}$ (blue crosses) and $M_{\text {err }}$ (red dashes).

Examining the center right and bottom right panels in Figure 4 shows that over most frequency ranges the $\mathbf{k}$ spectra fluctuate rapidly and the error parameter $\theta_{\text {err }}$ is large. For a small frequency range $2-2.4 \mathrm{~Hz}, M_{\text {err }} \leq 0.02$ and $\theta_{\text {err }} \simeq 45^{\circ}$, the angle $\theta=\tan ^{-1}\left(k_{y} / k_{x}\right)$ is stable, and the corresponding $k_{x}$ and $k_{y}$ spectra imply a perpendicular wavelength of $\sim 18 \mathrm{~km}$. However, because this frequency interval is so small and, unlike the previous example, does not correspond well to features in the power spectra, we have considerably less confidence in this result. On the other hand over the frequency range in which the spectral power is large $(3.7-5.4 \mathrm{~Hz})$, large error parameters suggest that in this case the condition that each frequency corresponds to a single wave vector may be violated.

The data from this interval do pass the less stringent internal check (Equation (5)) with values $\langle\mathbf{J}(t) \times \mathbf{B}(t)\rangle_{\text {norm }}=\left\langle 2.2 \times 10^{-3}, 1.8 \times 10^{-3}, 0\right\rangle$, which are small compared to unity. The lack of a clear result in Figure 4 suggests that this test alone is insufficient to determine whether the results of B16 analysis are reliable, and at minimum should be supplemented with calculation and examination of $\theta_{\text {err }}$ and $M_{\text {err }}$.

\section{Conclusions}

We have applied the recently published Bellan [2016] method to FAST observations of inertial Alfvén waves, where we are restricted to consideration of waves with spacecraftframe frequencies $f_{\mathrm{sp}} \lesssim 6 \mathrm{~Hz}$ because of sampling rate limitations of the ESAs aboard FAST. Through searching a database covering three years of observations, two promising intervals were identified in which the modulated parallel currents associated with the Alfvén waves were measurable with both the particle and magnetic field instruments. During the Jan. 23, 1999 interval, the analysis yields wave vector components corresponding to perpendicular wavelengths of approximately $2-12 \mathrm{~km}$ over a narrow frequency range corresponding to the peak spectral intensity of the current modulations and in which error parameters generated within the B16 analysis are small. These perpendicular wavelengths seem reasonable within the magnetosphere-ionosphere (M-I) transition region, where the local Alfvén speed is of order $10^{4} \mathrm{~km} / \mathrm{s}$ and the parallel wavelength at $1 \mathrm{~Hz}$ is therefore of order $10^{4} \mathrm{~km}$. During the Jan. 27, 1999 interval spectra show enhanced modulations in current density below $\sim 2 \mathrm{~Hz}$, but the analysis yielded no clear results for the estimated wave vector and relatively high error angles over that frequency range. It appears possible that although IAWs coincident with large current and field modulations occurred in this interval the conditions required for the analysis may not have held, in particular the requirement of a one-to-one relation between wave vector and frequency in the spacecraft frame.

This paper represents the first attempted application of the method prescribed by Bellan [2016] for inferring wave vectors from single-point measurements under restrictive conditions, to satellite observations in the M-I transition region. Gershman et al. [2017] have 
previously applied this method to MMS observations near the dayside magnetopause. In their application the properties of a monochromatic, kinetic-scale Alfvén wave are derived using four independent methods. From these results they adopt the wave vector calculated using the Bellan [2016] method because it incorporates measurements from all four Magnetospheric Multiscale spacecraft and all magnetic field components.

Our attempt suggests that the method could yield meaningful estimates of $\mathbf{k}$ given the proper combination of measurements of $\mathbf{B}$ and $\mathbf{J}$ and amenable physical conditions. In the data used here, only one component of the modulated current was measured. High quality measurements of all three components with sufficient cadence to resolve Alfvén waves remains an experimental challenge. Also left for future work is further testing of the method, through for example consideration of electric field measurements (a challenge with FAST because one component was not completely measured) or through comparison with spaced-probe interferometric measurements of $\mathbf{k}$.

Acknowledgments. We thank P. Bellan for making publicly available Interactive Data Language (IDL) routines for this analysis, which we have modified for use with FAST data. All FAST observations, along with a suite of Interactive Data Language analysis routines, have been obtained via the publicly available Satellite Data Tool (http://sprg.ssl. berkeley.edu/ sdt/SdtReleases.html). This work was supported by NASA NESSF fellowship NNX14AO03H, NASA grant NNX17AF92G to Dartmouth College, and subaward W000726838 from NASA grant NNX15AL08G. The Editors thank Ondrej Santolik and one anonymous reviewer for their help in evaluating this paper.

\section{References}

Bellan, P. M., Revised single-spacecraft method for determining wavevector k and resolving space-time ambiguity, J. Geophys. Res., 121, 8589-8599, 2016.

Bernstein, I. B., and F. Engelmann, Quasi-linear theory of plasma waves, Phys. Fluids, 9, 937-952, doi:10.1063/1.1761795, 1966.

Carlson, C. W., J. P. McFadden, P. Turin, D. W. Curtis, and A. Magoncelli, The electron and ion plasma experiment for FAST, Space Sci. Rev., 98, 1, 33-66, 2001.

Chaston, C. C., J. W. Bonnell, C. W. Carlson, J. P. McFadden, R. J. Strangeway, and R. E. Ergun, Kinetic effects in the acceleration of auroral electrons in small scale Alfvén waves: A FAST case study, Geophys. Res. Lett., 30, 6, id.1289, 2003.

Chaston, C. C., A. J. Hull, J. W. Bonnell, C. W. Carlson, R. E. Ergun, R. J. Strangeway, and J.P. McFadden, Large parallel electric fields, currents, and density cavities in dispersive Alfvén waves above the aurora, J. Geophys. Res., 112, A05215, doi:10.1029/2006JA012007, 2007.

Fredricks, R. W., and F. V. Coroniti, Ambiguities in the deduction of rest frame fluctuation spectrums from spectrums computed in moving frames, J. Geophys. Res., 81, 55915595, doi:10.1029/JA081i031p05591, 1976. 
Gershman, D. J., A. F-Viñas, J. C. Dorelli, S. A. Boardsen, L. A. Avanov, P. M. Bellan, S. J. Schwartz, B. Lavraud, V. N. Coffey, M. O. Chandler, Y. Saito, W. R. Paterson, S. A. Fuselier, R. E. Ergun, R. J. Strangeway, C. T. Russell, B. L. Giles, C. J. Pollock, R. B. Torbert, and J.L. Burch, Wave-particle energy exchange directly observed in a kinetic Alfvén-branch wave, Nature Communications, 8, id.14719, doi:10.1038/ncomms14719, 2017.

Goertz, C. K., and R. W. Boswell, Magnetosphere-ionosphere coupling, J. Geophys. Res., 84, 7239-7246, doi:10.1029/JA084iA12p07239, 1979.

Hatch, S. M., C. C. Chaston, and J. Labelle, Alfvén wave-driven ionospheric mass outflow and electron precipitation during storms, J. Geophys. Res., 121, 7828-7846, doi:10.1002/2016JA022805, 2016.

Hatch, S. M., J. LaBelle, W. Lotko, C. C. Chaston, and B. Zhang, IMF control of Alfvénic energy transport and deposition at high latitudes, J. Geophys. Res., 122, 12, 1218912211, 2017.

Hatch, S. M., J. LaBelle, and C. C. Chaston, Storm phase-partitioned rates and budgets of global Alfvénic energy deposition, electron precipitation, and ion outflow, J. Astr. Terr. Sol. Phys., 167, 1-12, 2018.

Keiling, A., Alfvén waves and their roles in the dynamics of the Earth's magnetotail: A review, Space Sci. Rev., 142, 73-156, doi:10.1007/s11214-008-9463-8, 2009.

Kletzing, C. A., F. S. Mozer, and R. B. Torbert, Electron temperature and density at high latitude, J. Geophys. Res., 103, A7, 14837-14845, doi:10.1029/98JA00962, 1998.

LaBelle, J., and P. M. Kintner, The measurement of wavelength in space plasmas, Rev. Geophys., 27, 4, 495-518, doi:10.1029/RG027i004p00495, 1989.

Pfaff, R.F., and P.A. Marionni, Multiple-baseline spaced receivers, in Meas. Tech. Sp. Plasmas Fields, American Geophysical Union, pp. 161-167, 1998.

Sandholt, P. E., C. J. Farrugia, and W. F. Denig, Detailed dayside auroral morphology as a function of local time for southeast IMF orientation: Implications for solar windmagnetosphere coupling, Ann. Geophys., 22, 10, 3537-3560, doi:10.5194/angeo-223537-2004, 2004.

Schmidt, G., IX - Nonlinear Waves, in Phys. High Temp. Plasmas (Second Ed.), Academic Press, doi:10.1016/B978-0-12-626660-3.50013-4, pp. 269-321, 1979.

Slepian, D., Prolate spheroidal wave functions, Fourier analysis, and uncertainty - V: The discrete case, Bell Syst. Tech. J., 57, 5, 1371-1430, 1978.

Slepian, D., Some comments on Fourier analysis, uncertainty and modeling, SIAM Rev., 25, 3, 379-393, doi:10.1137/1025078, 1983.

Stasiewicz, K., P. Bellan, C. Chaston, C. Kletzing, R. Lysak, J. Maggs, O. Pokhotelov, C. Seyler, P. Shukla, L. Stenflo, A. Streltsov, and J.-E. Wahlund, Small scale Alfvénic structure in the aurora, Space Sci. Rev., 92, 423-533, 2000. 
